\title{
Is high-flow safer than low-flow nasal oxygenation for procedural sedation?
}

\author{
Mahesh Nagappa, MD (i) - David T. Wong, MD
}

Received: 5 November 2020/Revised: 16 November 2020/Accepted: 17 November 2020/Published online: 12 January 2021

(C) Canadian Anesthesiologists' Society 2021

Each year, millions of patients in North America undergo gastrointestinal endoscopic procedures under propofol sedation without serious sequelae. The most common complication is transient non-life-threatening oxygen desaturation, which can be managed and reversed by airway maneuvers and/or adjusting the depth of sedation. Oxygen desaturation during a procedure is a surrogate outcome, but some important "hard" outcomes with patient and economic consequences include tracheal intubation, delayed ambulatory discharge, or unanticipated hospital admission. In the literature, different outcomes have been used to define "desaturation" as an adverse outcome. Some authors use a pulse oximetry $\left(\mathrm{SpO}_{2}\right)$ cutoff of $92 \%$ to define desaturation, while others may use $85 \% .^{1}$ In this issue of the Journal, Kim et al. ${ }^{2}$ used a $\mathrm{SpO}_{2}$ cutoff of $90 \%$ to define desaturation. Irrespective of the cutoff values, all grades of hypoxia come under one umbrella term "desaturation".

Kim et al. compared two methods of oxygen delivery in patients undergoing endoscopic retrograde cholangiopancreatography, which is a gastrointestinal endoscopic procedure commonly performed under deep sedation in the prone position. The authors randomized

\footnotetext{
M. Nagappa, MD ( $\varangle)$

Department of Anesthesia \& Perioperative Medicine, London Health Sciences Centre and St. Joseph Health Care, Western University, Schulich School of Medicine and Dentistry, London, ON, Canada

e-mail: Mahesh.Nagappa@lhsc.on.ca

D. T. Wong, MD

Department of Anesthesiology and Pain Medicine, Toronto Western Hospital, University Health Network, University of Toronto, Toronto, ON, Canada
}

patients to receive oxygen using either high-flow nasal oxygen (HFNO) or conventional nasal cannula. The primary outcome of the lowest oxygen saturation measured via pulse oximetry during the procedure was found to be higher in the HFNO group than in the conventional nasal cannula group (99.8 vs $95.1 \%$ ). The secondary outcomes of incidence of hypoxemia (defined as desaturation $<90 \%$ [0\% vs $19.4 \%]$ ), duration of hypoxemia ( 0 vs $17.4 \mathrm{sec})$, procedure interruptions due to airway intervention like discontinuing sedation (0 vs 25\%), patient stimulation ( 0 vs 27.8\%), jaw thrust maneuver ( 0 vs $27.8 \%)$. and end-tidal $\mathrm{CO}_{2}(30.4$ vs $33.9 \mathrm{mmHg}$ ) at the end of the procedure were also better in the HFNO group.

Even though the lowest $\mathrm{SpO}_{2}$ recorded in the conventional nasal cannula group was significantly lower than in the HFNO group, an oxygen saturation of $95.1 \%$ is quite acceptable in the clinical setting. Moreover, the incidence of hypoxemia of $19 \%$ for a short period of $17 \mathrm{sec}$ may not be considered a clinically significant outcome. Nevertheless, the authors provided a novel and impactful clinical insight by showing that HFNO can provide excellent oxygen delivery and adequate oxygenation without any airway intervention in this challenging clinical context. The authors suggest that during this procedure, HFNO may be considered a safe, standard oxygen delivery method as compared with the use of conventional nasal cannula.

The findings of Kim et al. are consistent with the results of a recently published meta-analysis ${ }^{3}$ which showed that the use of HFNO increased patient safety with significant reduction in episodes of oxygen desaturation, as well as the need for airway intervention. The meta-analysis included three randomized-controlled trials with 2,113 patients undergoing procedural sedation, where 1,052 patients received HFNO and 1,061 patients received conventional 
oxygen. The odds ratio of desaturation for the HFNO group compared with the control group was found to be 0.09 (95\% confidence interval [CI], 0.05 to 0.18 ; HFNO vs control incidence: $1.4 \%$ vs $10 \%){ }^{3}$ Similarly, the minimum (standard deviation) oxygen saturation was higher in the HFNO group than in the control group [93\% (6) vs $89 \%$ (5); mean difference, 3.9 ; $95 \% \mathrm{CI}, 1.7$ to 6.1$] .{ }^{3}-^{5}$ Furthermore, the use of HFNO prolonged the safe apnea time - which is very important to the anesthesiologist during induction - by $33.4 \mathrm{sec}$ compared with the use of standard oxygen delivery (95\% CI, 16.8 to 50.1$).{ }^{3,6}{ }^{8}$

The physiologic effect of HFNO and its mechanism of action has several advantages over traditional nasal cannula. The use of HFNO supplies warm humidified oxygen through nasal prongs at high flow rates, ranging between 40 and $70 \mathrm{~L} \cdot \mathrm{min}^{-1}$. 9 The fraction of inspired oxygen $\left(\mathrm{F}_{1} \mathrm{O}_{2}\right)$ can reach up to $100 \% .^{10,11}$ Unlike the conventional method of oxygenation (face mask or nasal cannula), HFNO can provide oxygen flow rates that are equal to or higher than the patients' inspiratory flow rate. This reduces or eliminates the entrainment of room air during inspiratory effort, and thereby reliably maintains a high $\mathrm{F}_{1} \mathrm{O}_{2} \cdot{ }^{9}-{ }^{11}$ The HFNO reduces the rate of accumulation of the partial pressure of carbon dioxide by washing out and reducing the anatomical dead space. In addition, for every $10 \mathrm{~L} \cdot \mathrm{min}^{-1}$ of oxygen flow, HFNO provides approximately $1 \mathrm{~cm}$ of $\mathrm{H}_{2} \mathrm{O}$ of continuous positive airway pressure, which improves oxygenation. ${ }^{10}{ }^{12}$ Overall, HFNO reduces the patient's work of breathing and enhances the patient's lung compliance.

That said, there are a few potential downsides to using HFNO compared with nasal cannula. In addition to limited availability and higher cost, one of the major downsides with HFNO is lack of capnography monitoring in a dark, high risk environment in a remote endoscopy suite, away from the operating room. Indeed, the Canadian Anesthesiologists' Society practice guidelines ${ }^{13}$ and other international societies' guidelines ${ }^{14}{ }^{17}$ mandate continuous waveform capnography for patients expected to require moderate or deep procedural sedation. Two important monitoring devices during sedation are pulse oximetry and capnography, which are designed to measure two different physiologic processes, namely oxygenation and ventilation. Continuous capnography can detect apnea, bradypnea, or hypercarbia, while continuous pulse oximetry detects oxygen desaturation. In addition, capnography can identify and differentiate upper airway obstruction, laryngospasm, and bronchospasm. ${ }^{18}{ }^{23}$ Apneic episodes precede desaturation by $60 \mathrm{sec}$ (range, $5-240 \mathrm{sec}$ ) and the absence of capnography during sedation may carry the risk of delaying the detection of apnea. ${ }^{24}$ Moreover, oxygen in the patient's lung enriched by HFNO may further delay detection of apnea by rendering pulse oximetry ineffective as an early warning device for hypoventilation. Therefore, capnography is strongly recommended when additional oxygen is provided, as capnography readings are not affected by the presence or absence of extra oxygen. In the study by Kim et al., the high $\mathrm{F}_{1} \mathrm{O}_{2}$ in $\mathrm{HFNO}$ and good $\mathrm{SpO}_{2}$ could have masked possible apneic episodes that occurred in the HFNO group, but that were picked up in the nasal cannula group, prompting airway maneuvers/sedation changes.

Traditionally, HFNO has not had capnography "builtin", but this is beginning to change. Greenland successfully used a 14-G cannula placed in the oxygen inlet of the Oxyguard $^{\mathrm{TM}}$ bite block to capture a capnography waveform during HFNO administration. ${ }^{25}$ A new Optiflow THRIVE ${ }^{\mathrm{TM}}$ nasal high-flow cannula with an integrated capnography sampling tube has recently been developed - Optiflow Trace ${ }^{\mathrm{TM}}$ (Fisher and Paykel Healthcare, Panmure, Auckland, New Zealand). In that system, exhaled gases are analyzed via the integrated $\mathrm{CO}_{2}$ sampling interface, which can be configured to sample gas either from the mouth or from the nose. Nevertheless, the absolute end-tidal $\mathrm{CO}_{2}\left(\mathrm{EtCO}_{2}\right)$ values reported when using the HFNO may be significantly lower than the actual value. Nevertheless, this potential inaccuracy of the absolute $\mathrm{EtCO}_{2}$ value is likely inconsequential since the primary value of waveform capnography in this context is the benefit of being able to see the breath-to-breath $\mathrm{CO}_{2}$ waveform, which allows for the rapid diagnosis of apnea or airway obstruction.

Technical problems, lack of familiarity, and cost may limit the use of HFNO in various clinical conditions. Despite these challenges, the patient safety benefits of HFNO to prevent hypoxemia may offset these challenges, as more and more patients with higher risk of desaturation are encountered in current medical practice. Patients with morbid obesity, respiratory insufficiency, undergoing bronchoscopy or awake intubation, history of difficulty airway, rapid sequence intubation, and difficult sedation procedures are some of the broad range of clinical applications for HFNO.

In closed claim reviews related to sedation procedures outside the operating room, it was found that inadequate oxygenation and inadequate ventilation are the most frequent events leading to the median cost claim of USD $330,000 .^{26,27}$ The superior oxygenation and ventilation provided by HFNO may reduce such claims. Large, welldesigned randomized-controlled trials are required to confirm the direct causal relationship between the use of HFNO and reduction of patient harm (e.g., intubation complications, delayed discharge, hospital admission, myocardial infarction, and death). Nevertheless, it is important to understand that such trials may not be feasible as thousands of patients would be required. As 
well, looking for this type of evidence may delay the broader acceptance of HFNO as an important device for better oxygenation/ventilation and improved safety. So, we are left with accepting HFNO as better than nasal cannula in procedural sedation for improved surrogate outcomes, but not yet for important hard outcomes. At this time, we recommend HFNO with capnography during procedural sedation in higher risk patient populations with underlying pulmonary conditions or morbid obesity, but not yet for routine use in lower risk patients.

\section{Sédation procédurale: l'oxygénation nasale à débit élevé est-elle plus sécuritaire que l'oxygénation nasale à faible débit?}

Chaque année, des millions de patients en Amérique du Nord subissent des interventions endoscopiques gastrointestinales sous sédation au propofol sans séquelles graves. La complication la plus fréquente est une désaturation en oxygène transitoire ne mettant pas la vie en danger, qui peut être prise en charge et adressée par des manipulations au niveau des voies aériennes et/ou en ajustant la profondeur de la sédation. La désaturation en oxygène au cours d'une intervention constitue un résultat intermédiaire, mais certains résultats cliniques importants (ou 'hard outcome' en anglais) s'accompagnent de conséquences économiques et pour les patients, notamment l'intubation trachéale, le retard dans l'obtention du congé, ou une admission imprévue à l'hôpital. Dans la littérature, différents critères ont été utilisés pour définir la « désaturation » comme un devenir défavorable. Certains auteurs utilisent un seuil d'oxymétrie de pouls $\left(\mathrm{SpO}_{2}\right)$ de $92 \%$ pour définir la désaturation, tandis que d'autres utilisent un seuil de $85 \%{ }^{1}$ Dans ce numéro du Journal, Kim et coll. ${ }^{2}$ se sont fondés sur un seuil de $\mathrm{SpO}_{2}$ à $90 \%$ pour définir la désaturation. Indépendamment des valeurs retenues, tous les niveaux d'hypoxie ont été regroupés sous un seul terme générique de « désaturation».

Kim et coll. ont comparé deux méthodes d'oxygénation chez des patients subissant une cholangiopancréatographie rétrograde endoscopique, une intervention endoscopique gastro-intestinale généralement exécutée sous sédation profonde en position ventrale. Les auteurs ont randomisé les patients à recevoir l'oxygène soit par canule nasale à haut débit (CNHD), ou par canule nasale conventionnelle. Le critère d'évaluation principal, soit la plus faible saturation en oxygène mesurée par oxymétrie de pouls pendant l'intervention, s'est révélé plus élevé dans le groupe CNHD que dans le groupe de canule nasale conventionnelle (99,8 vs $95,1 \%)$. Les critères d'évaluation secondaires, soit l'incidence d'hypoxémie (définie comme une désaturation $<90 \%[0 \%$ vs $19,4 \%]$ ), la durée de l'hypoxémie (0 vs 17,4 sec), les interruptions de l'intervention dues à des interventions au niveau des voies respiratoires comme un arrêt de sédation ( 0 vs $25 \%$ ), la stimulation du patient (0 vs 27,8\%), la subluxation de la mâchoire ( 0 vs $27,8 \%)$ et la valeur téléexpiratoire du $\mathrm{CO}_{2}(30,4$ vs $33,9 \mathrm{mmHg})$ à la fin de l'intervention étaient également meilleurs dans le groupe CNHD.

Même si la $\mathrm{SpO}_{2}$ la plus basse enregistrée dans le groupe canule nasale conventionnelle était sensiblement inférieure que dans le groupe CNHD, une saturation en oxygène de $95,1 \%$ est tout à fait acceptable dans un cadre clinique. En outre, l'incidence d'hypoxémie de $19 \%$ pendant une courte période de 17 secondes peut ne pas être considérée comme un résultat cliniquement significatif. Néanmoins, les auteurs proposent une perspective clinique novatrice et perspicace en montrant qu'une canule nasale à haut débit peut procurer un excellent apport en oxygène et une oxygénation adéquate sans aucune intervention au niveau des voies aériennes dans ce type de contexte clinique difficile. Les auteurs suggèrent que pendant cette intervention, la CNHD peut être considérée comme une méthode sécuritaire et standard d'oxygénation par rapport à l'utilisation d'une canule nasale conventionnelle.

Les conclusions de Kim et coll. sont en adéquation avec les résultats d'une méta-analyse récemment publiée ${ }^{3}$ qui a montré que l'utilisation d'une CNHD augmentait la sécurité des patients avec une réduction significative des épisodes de désaturation en oxygène, ainsi que de la nécessité des interventions au niveau des voies respiratoires. La méta-analyse comprenait trois études randomisées contrôlées avec 2113 patients subissant une sédation procédurale, où 1052 patients ont reçu une CNHD et 1061 patients ont reçu de l'oxygène par voie conventionnelle. Le rapport de cotes pour la désaturation dans le groupe CNHD par rapport au groupe témoin était de 0,09 (intervalle de confiance [IC] $95 \%, 0,05$ à 0,18 ; incidence dans le groupe CNHD vs témoin : $1,4 \% v s$ $10 \%){ }^{3}$ De même, la saturation en oxygène minimale (écart type) était plus élevée dans le groupe CNHD que dans le groupe témoin [93\% (6) vs $89 \%$ (5); différence moyenne, 3,9; IC $95 \%, 1,7$ à 6,1$].{ }^{3}{ }^{5}{ }^{5}$ En outre, l'utilisation d'une CNHD a prolongé le temps d'apnée sécuritaire lequel est très important pour l'anesthésiologiste pendant l'induction - de 33,4 secondes par rapport à l'utilisation d'une livraison standard d'oxygène (IC 95\%, 16,8 à $50,1){ }^{3,6}{ }^{8}$ 
L'effet physiologique de la CNHD et son mécanisme d'action présentent plusieurs avantages par rapport aux canules nasales traditionnelles. L'utilisation d'une CNHD fournit de l'oxygène humidifié chaud par de courtes tiges nasales à des débits élevés, allant de 40 à $70 \mathrm{~L} \cdot \mathrm{min}^{-1}{ }^{9}{ }^{9} \mathrm{La}$ fraction d'oxygène inspiré $\left(\mathrm{F}_{1} \mathrm{O}_{2}\right)$ peut atteindre jusqu'à $100 \%{ }^{10,11}$ Contrairement à la méthode conventionnelle d'oxygénation (par masque facial ou canule nasale), une CNHD peut procurer des débits d'oxygène égaux ou supérieurs au débit inspiratoire des patients. Cela réduit ou élimine l'entraînement de l'air ambiant pendant l'effort inspiratoire, et maintient ainsi de manière fiable une $\mathrm{F}_{1} \mathrm{O}_{2}$ élevée. ${ }^{9}{ }^{11}$ La CNHD réduit le taux d'accumulation du dioxyde de carbone en l'éliminant du et en réduisant l'espace mort anatomique. En outre, pour chaque $10 \mathrm{~L} \cdot \mathrm{min}^{-1}$ de débit d'oxygène, la CNHD procure environ $1 \mathrm{~cm}$ de $\mathrm{H}_{2} \mathrm{O}$ de pression positive continue, ce qui améliore l'oxygénation. ${ }^{10}{ }^{12}$ Dans l'ensemble, les CNHD réduisent le travail de respiration du patient et améliorent sa compliance pulmonaire.

Cela dit, il existe quelques inconvénients potentiels à l'utilisation d'une CNHD par rapport à une canule nasale. Outre sa disponibilité limitée et son coût plus élevé, l'un des principaux inconvénients de la CNHD est le manque de monitorage par capnographie dans un environnement sombre et à haut risque dans une salle d'endoscopie éloignée, loin de la salle d'opération. En effet, le Guide d'exercice de la Société canadienne des anesthésiologistes $^{13}$ et les lignes directrices d'autres sociétés internationales ${ }^{14}{ }^{17}$ exigent une capnographie continue pour les patients pour lesquels une sédation procédurale modérée ou profonde est prévue. Deux dispositifs de monitorage importants pendant la sédation sont l'oxymétrie de pouls et la capnographie, lesquels sont conçus pour mesurer deux processus physiologiques différents, à savoir l'oxygénation et la ventilation. La capnographie en continu peut détecter une apnée, une bradypnée ou une hypercapnie, tandis que l'oxymétrie de pouls en continu détectera la désaturation en oxygène. En outre, la capnographie peut identifier et différencier une obstruction des voies aériennes supérieures, un laryngospasme et un bronchospasme. ${ }^{18}{ }^{23}$ Les épisodes apnéiques précèdent la désaturation de $60 \mathrm{sec}$ (portée, 5-240 sec); l'absence de capnographie pendant la sédation peut entrainer le risque de retarder la détection d'une apnée. ${ }^{24}$ En outre, l'oxygène dans le poumon du patient enrichi par la CNHD peut retarder davantage la détection d'une apnée en rendant l'oxymétrie de pouls inefficace en tant que dispositif d'alerte précoce d'une hypoventilation. Par conséquent, la capnographie est fortement recommandée lorsque de l'oxygène supplémentaire est fourni, car elle n'est pas affectée par la présence ou l'absence d'oxygène supplémentaire. Dans l'étude de Kim et coll., la $\mathrm{F}_{\mathrm{I}} \mathrm{O}_{2}$ élevée fournie par une CNHD et une bonne $\mathrm{SpO}_{2}$ auraient pu masquer les épisodes apnéiques possibles qui se sont produits dans le groupe CNHD, mais qui ont été remarqués dans le groupe de canule nasale, causant des manœuvres au niveau des voies aériennes ou des changements de sédation.

Traditionnellement, les CNHD n'ont pas de capnographie « intégrée », mais les modèles commencent à évoluer. Greenland a utilisé avec succès une canule de 14 G placée dans l'entrée d'oxygène de la pièce buccale Oxyguard $^{\mathrm{TM}}$ pour obtenir une capnographie avec l'utilisation d'une CNHD. ${ }^{25}$ Une nouvelle canule nasale à haut débit Optiflow THRIVE ${ }^{\mathrm{TM}}$ avec un tube d'échantillonnage capnographique intégré a récemment été mise au point, l'Optiflow Trace ${ }^{\mathrm{TM}}$ (Fisher and Paykel Healthcare, Panmure, Auckland, Nouvelle-Zélande). Dans ce système, les gaz expirés sont analysés par l'intermédiaire de l'interface intégrée d'échantillonnage du $\mathrm{CO}_{2}$, qui peut être configurée pour échantillonner les gaz de la bouche ou du nez. Néanmoins, les valeurs absolues de pression télé-expiratoire en $\mathrm{CO}_{2}\left(\mathrm{EtCO}_{2}\right)$ rapportées lors de l'utilisation d'une CNHD peuvent être significativement inférieures à la valeur réelle. Ceci étant dit, cette inexactitude potentielle de la valeur absolue d'EtCO ${ }_{2}$ est probablement sans conséquence. En effet, l'intérêt principal de la capnographie dans ce contexte est l'avantage de pouvoir voir la forme d'onde de $\mathrm{CO}_{2}$ à chaque respiration, ce qui permet de poser rapidement un diagnostic d'apnée ou d'obstruction des voies aériennes.

Les problèmes techniques, le manque de familiarité et le coût pourraient limiter l'utilité des CNHD dans diverses conditions cliniques. En dépit de ces défis, les avantages en matière de sécurité des patients des CNHD pour prévenir l'hypoxémie pourraient compenser ces difficultés, alors qu'un nombre toujours croissant de patients présentant un risque plus élevé de désaturation sont rencontrés dans la pratique médicale actuelle. Les patients présentant une obésité morbide, une insuffisance respiratoire, subissant une bronchoscopie ou une intubation éveillée, ayant des antécédents de voies aériennes difficiles, d'intubation en séquence rapide, et d'intervention difficiles sous sédation sont quelques-unes des nombreuses applications cliniques des CNHD.

Dans les examens des réclamations légales réglées liées aux interventions de sédation à l'extérieur de la salle d'opération, il a été constaté qu'une oxygénation et une ventilation inadéquates étaient les événements les plus fréquents, menant à un coût médian de réclamation de 330 000 USD. ${ }^{26,27}$ L'oxygénation et la ventilation supérieures fournies par une CNHD pourraient réduire ces réclamations. De grandes études randomisées contrôlées bien conçues sont nécessaires pour confirmer la relation causale directe entre l'utilisation d'une CNHD et la 
réduction des torts causés aux patients (p. ex., complications liées à l'intubation, congé retardé, admission à l'hôpital, infarctus du myocarde, et décès). Néanmoins, il est important de comprendre que de telles études pourraient ne pas être réalisables, car des milliers de patients seraient nécessaires. De plus, la quête pour ce type de données probantes pourrait retarder l'acceptation plus répandue des CNHD comme un dispositif important pour une meilleure oxygénation/ventilation et une sécurité améliorée. Ainsi, il ne nous reste qu'à accepter la CNHD comme étant supérieure à la canule nasale pour la sédation procédurale pour améliorer les résultats intermédiaires, mais pas encore pour améliorer les résultats quantifiables importants. À l'heure actuelle, nous recommandons d'utiliser les CNHD avec la capnographie pendant la sédation procédurale dans les populations de patients à risque plus élevé présentant des conditions pulmonaires sous-jacentes ou une obésité morbide, mais pas encore pour une utilisation courante chez les patients à faible risque.

Disclosures None.

\section{Funding statement None.}

Editorial responsibility This submission was handled by Dr. Philip M. Jones, Associate Editor, Canadian Journal of Anesthesia.

Déclaration Aucune.

Déclaration de financement Aucune.

Responsabilite' e'ditoriale Cet article a été traité par Dr Philip M. Jones, rédacteur adjoint, Journal canadien d'anesthésie.

\section{References}

1. Saunders $R$, Struys MM, Pollock RF, Mestek M, Lightdale JR. Patient safety during procedural sedation using capnography monitoring: a systematic review and meta-analysis. BMJ Open 2017; DOI: https://doi.org/10.1136/bmjopen-2016-013402.

2. Kim SH, Bang S, Lee KY, et al. Comparison of high flow nasal oxygen and conventional nasal cannula during gastrointestinal endoscopic sedation in the prone position: a randomized trial. Can J Anesth 2021; DOI: https://doi.org/10.1007/s12630-02001883-2.

3. Spence EA, Rajaleelan W, Wong J, Chung F, Wong DT. The effectiveness of high-flow nasal oxygen during the intraoperative period: a systematic review and meta-analysis. Anesth Analg 2020; 131: 1102-10.

4. Douglas $N, N g I$, Nazeem F et al. A randomised controlled trial comparing high-flow nasal oxygen with standard management for conscious sedation during bronchoscopy. Anaesthesia 2018; 73 : 169-76.

5. Takakuwa $O$, Oguri T, Asano T, et al. Prevention of hypoxemia during endobronchial ultrasound-guided transbronchial needle aspiration: usefulness of high-flow nasal cannula. Respir Investig 2018; 56: 418-23.
6. Lodenius A, Piehl J, Östlund A, Ullman J, Jonsson Fagerlund M. Transnasal humidified rapid-insufflation ventilatory exchange (THRIVE) vs. facemask breathing pre-oxygenation for rapid sequence induction in adults: a prospective randomised nonblinded clinical trial. Anaesthesia 2018; 73: 564-71.

7. Wong DT, Dallaire A, Singh KP, et al. High-flow nasal oxygen improves safe apnea time in morbidly obese patients undergoing general anesthesia: a randomized controlled trial. Anesth Analg 2019; 129: 1130-6.

8. Mir F, Patel A, Iqbal R, Cecconi M, Nouraei SA. A randomised controlled trial comparing transnasal humidified rapid insufflation ventilatory exchange (THRIVE) pre-oxygenation with facemask pre-oxygenation in patients undergoing rapid sequence induction of anaesthesia. Anaesthesia 2017; 72: 439-43.

9. Jaber S, Monnin M, Girard M, et al. Apnoeic oxygenation via high-flow nasal cannula oxygen combined with non-invasive ventilation preoxygenation for intubation in hypoxaemic patients in the intensive care unit: the single-centre, blinded, randomised controlled OPTINIV trial. Intensive Care Med 2016; 42: 1877-87.

10. Wong DT, Yee AJ, Leong SM, Chung F. The effectiveness of apneic oxygenation during tracheal intubation in various clinical settings: a narrative review. Can J Anesth 2017; 64: 416-27.

11. Helviz $Y$, Einav $S$. A systematic review of the high-flow nasal cannula for adult patients. Crit Care 2018; DOI: https://doi.org/ 10.1186/s13054-018-1990-4.

12. Spoletini G, Hill NS. High-flow nasal oxygen versus noninvasive ventilation for hypoxemic respiratory failure: do we know enough? Ann Thorac Med 2016; 11: 163-6.

13. Dobson G, Chow L, Filteau L, et al. Guidelines to the practice of anesthesia - revised edition 2020. Can J Anesth 2020; 67: 64-99.

14. Association of Anaesthetists of Great Britain and Ireland (AAGBI). AAGBI Safety Statement. The use of capnography outside the operating theatre. Available from URL: https://static1. squarespace.com/static/54d14bfce4b02b4744e70d6d/t/ 54f5ff77e4b0eaa8361e1e4e/1425407863573/ AAGBI+Safety+Statement+5_12.pdf (accessed November 2020).

15. Checketts MR, Alladi R, Ferguson $K$, et al. Recommendations for standards of monitoring during anaesthesia and recovery 2015: Association of Anaesthetists of Great Britain and Ireland. Anaesthesia 2016; 71: 85-93.

16. The Australian and New Zealand College of Anaesthetists (ANZCA). Guidelines on Monitoring During Anaesthesia. Available from URL: https://www.anzca.edu.au/getattachment/ 0c2d9717-fa82-4507-a3d6-3533d8fa844d/PS18-Guideline-onmonitoring-during-anaesthesia (accessed November 2020).

17. American Society of Anesthesiologists. Standards for basic anesthetic monitoring. Available from URL: https://www.asahq. org/-/media/sites/asahq/files/public/resources/standardsguidelines/standards-for-basic-anesthetic-monitoring.pdf (accessed November 2020).

18. Krauss B. Capnography as a rapid assessment and triage tool for chemical terrorism. Pediatr Emerg Care 2005; 21: 493-7.

19. Coté CJ, Wax DF, Jennings MA, Gorski CL, Kurczak-Klippstein $K$. Endtidal carbon dioxide monitoring in children with congenital heart disease during sedation for cardiac catheterization by nonanesthesiologists. Paediatr Anaesth 2007; 17: 661-6.

20. Krauss BS, Andolfatto G, Krauss BA, Mieloszyk RJ, Monuteaux $M C$. Characteristics of and predictors for apnea and clinical interventions during procedural sedation. Ann Emerg Med 2016; 68: 564-73.

21. Miner JR, Heegaard W, Plummer D. End-tidal carbon dioxide monitoring during procedural sedation. Acad Emerg Med 2002; 9: $275-80$. 
22. Burton JH, Harrah JD, Germann CA, Dillon DC. Does end-tidal carbon dioxide monitoring detect respiratory events prior to current sedation monitoring practices? Acad Emerg Med 2006; 13: $500-4$.

23. Lightdale JR, Goldman DA, Feldman HA, Newburg AR, DiNardo $J A$, Fox VL. Microstream capnography improves patient monitoring during moderate sedation: a randomized, controlled trial. Pediatrics 2006; 117: e1170-8.

24. Deitch K, Miner J, Chudnofsky CR, Dominici P, Latta D. Does end tidal $\mathrm{CO} 2$ monitoring during emergency department procedural sedation and analgesia with propofol decrease the incidence of hypoxic events? A randomized, controlled trial. Ann Emerg Med 2010; 55: 258-64.
25. Greenland $K B$. A potential method for obtaining wave-form capnography during high flow nasal oxygen. Anaesth Intensive Care 2019; 47: 204-6.

26. Metzner J, Posner KL, Domino KB. The risk and safety of anesthesia at remote locations: the US closed claims analysis. Curr Opin Anaesthesiol 2009; 22: 502-8.

27. Robbertze R, Posner KL, Domino KB. Closed claims review of anesthesia for procedures outside the operating room. Curr Opin Anaesthesiol 2006; 19: 436-42.

Publisher's Note Springer Nature remains neutral with regard to jurisdictional claims in published maps and institutional affiliations. 